to enclose their local cells. In the f.c.c. packing, the second layer has 42 spheres. In the related hexagonal close packing, it has 44, giving a total of 57 spheres when the inner 13 are included. Thus up to $57 \times 3-6=165$ parameters are needed to specify all configurations of two layers, making their classification and analysis a formidable task.

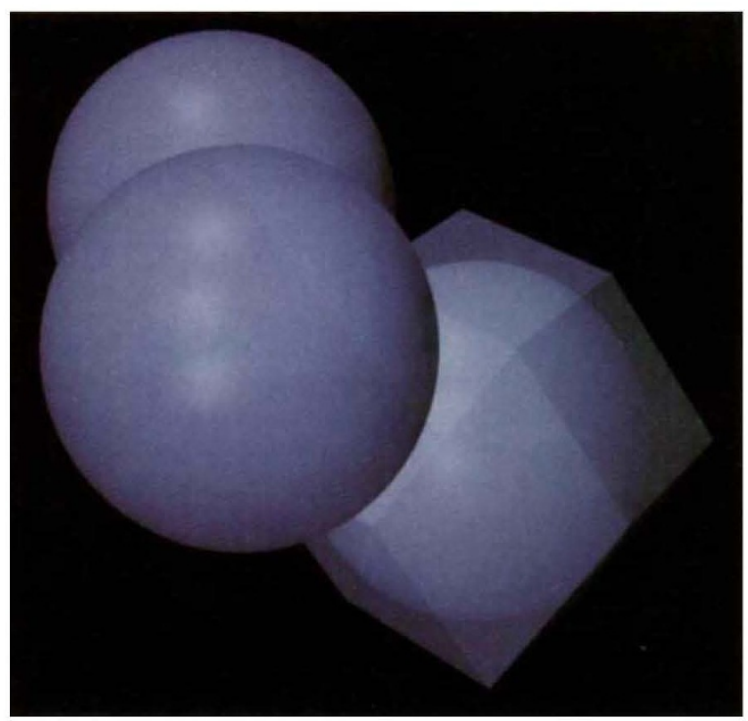

FIG. 2 The local cell for a sphere in f.c.c. packing. The 12 surrounding spheres are centred on the midpoints of the sides of the cube of side $2 \sqrt{ } 2$ (two are shown).

Hsiang's strategy was to eliminate most configurations as giving much too large a volume, and then to show that the remaining possibilities are close to one of a few key cases, like those mentioned above, which are analysed more carefully. It is this classification that takes up most of the second, longer preprint ${ }^{5}$, and it took Hsiang over a year to organize. Although he gives some explicit constants to separate the cases, many distinctions in the current draft are described qualitatively. This makes for easier reading but harder verification. Hsiang decided that to include the full details would make a much longer and more boring paper.

What is the current status of the proof? The original announcement, submitted to the Bulletin of the American Mathematical Society late in 1990, was rejected because the detailed proof had not yet been written down, but now it has. The draft will be circulated for comments to interested mathematicians for a few months. Hsiang is also presenting the details to the students in his graduate mathematics class. The current draft is the second, and it will probably be revised again before submission for publication. I have already pointed out a couple of minor, easily corrected misstatements, and presumably others will suggest improvements. But I doubt anyone will have the patience to repeat Hsiang's year-long verification that all possibilities are covered.

Why not do the detailed verification by computer, as in the four-colour map theorem? This does not seem easy. The four-colour problem was basically a combinational one, whereas the packing problem involves a large number of real position variables and a careful choice of technical parameters to distinguish the cases. Hsiang did not use a computer at all, although one sits in his office. He did the many spherical trigonometry and volume calculations on his trusty TI $35+$ pocket calculator.

And the future? Hsiang is already working on the four-dimensional case. There is a well-known lattice packing called $\mathrm{D}_{4}$, in which each sphere is surrounded by 24 touching neighbours. Hsiang claims to have a proof that this is the only configuration in which 24 spheres can touch a central one. He conjectures that this $\mathrm{D}_{4}$ packing will have the minimum local cell volume. This would give it the maximum density, as in the twodimensional case, because it is a lattice packing.

As for applications to the physical sciences, Hugh DeWitt at the Lawrence Livermore National Laboratory has written to Hsiang to recommend "a very similar problem in three dimensional space that is important in physics right now - the lowest possible energy of point charges confined in a finite volume, and with a uniform fixed background of opposite charge to give overall charge neutrality. This system is called the One Component Plasma. It has very important applications as a model for the interior of white dwarf stars and to some terrestrial systems as well .... the b.c.c. [body-centred cubic] lattice is very slightly lower in energy than the f.c.c. lattice in contrast to the hard sphere problem. As with the hard sphere packing problem, all physicists are convinced that the b.c.c. lattice is the lowest energy state, but nobody knows how to prove it".

Nelson Max is in the Department of Applied Science, University of California, Davis, California 95616, USA.

1. Kepier, J. On the Six Cornered Snowflake (Oxford University Press, 1966).

2. Hsiang, W. Bull. Brazilian math. Soc. (in the press)

3. Hilbert, D. Archiv. Math. Phys 1, 44-63 (1901)

4. Gauss, C. Werke II 188-196 (1876)

5. Hsiang, W. Reports PAM-530; PAM-535 (Center fo Pure and Applied Mathematics, University of California Berkeley, 1991).

6. Hsiang, W. Report PAM-536 (Center for Pure and Applied Mathematics, University of California, 1991).

\section{Artesian diver}

INDUSTRIAL society produces an everincreasing quantity of rubbish. Burning it in air, even in specialized furnaces, gives rise to air pollution. Daedalus now proposes to burn it in water instead.

This process is already used to destroy some specialized chemical wastes. They are dispersed in oxygen-bearing water which is heated to criticality at $380^{\circ} \mathrm{C}$ and 220 atmospheres pressure. Supercritical fluids are powerful solvents and reaction media. The organic material dissolves and oxidizes flamelessly and completely; the products remain safely in solution. To scale this process up would stretch high-pressure engineering to its limits. But Daedalus is undaunted. He suggests sinking an artesian well $2.2 \mathrm{~km}$ deep, when the hydrostatic pressure at the bottom will reach 220 atmospheres.

The necessary temperature of $380{ }^{\circ} \mathrm{C}$ needs a bit more cunning. Daedalus would like to use deep geothermal heat, thus achieving both pressure and temperature naturally; but in most places geology is against him. Instead, he recalls a scheme for keeping a diver warm in the icy ocean by beaming microwaves down his air pipe, which is configured as a wave guide. So by pumping air down an artesian well through a pipe which also acts as a wave guide, copious microwave heating could be provided at the bottom. The water there should rapidly reach supercriticality. Even better, microwaves promote chemical reactions wonderfully. Many traditional laboratory processes are greatly accelerated by microwave heating. Oxygenated, supercritical, microwave-heated water should dissolve and burn all forms of waste material rapidly and completely.

So Daedalus's artesian waste-combustor consists simply of two artesian wells connected at the bottom. Air, microwaves and every kind of rubbish and sewage sludge will be pumped down one of them; steam, carbon dioxide and hot water will fizz violently out of the other, providing copious heat and power. Even allowing for the power fed back to generate the microwaves, the installation will make a splendid energetic profit.

Under the ferocious conditions at the bottom, combustion will be guaranteed complete. All organic material will go to carbon dioxide, steam and nitrogen; minerals will be decomposed and equilibrated; the chlorine in polychlorinated biphenyls and PVC will be perfectly disengaged with no parts per billion of dioxin or chloroform to worry environmentalists. The outflow water will be utterly sterile, subtly mineralized and pleasantly carbonated. If its origin can be kept quiet, it should outsell the smartest mineral waters.

David Jones 